\title{
Die Lebenszeit des Dichters Romanos.
}

Im Anhange eines Aufsatzes über Umarbeitungen bei Romanos in den Sitzungsberichten der philos.-philol. und histor. Klasse der Kgl. Bayer. Akademie d. W. 1899, Bd. II p. 1 ff. äufsert sich Krumbacher auch über die Lebenszeit des Dichters, von dem wir nur wissen, dals er zur Zeit eines Kaisers Anastasios nach Byzanz kam, ohne dals hinzugefügt wird, welcher der beiden Kaiser dieses Namens gemeint sei. Während Krumbacher in der Gesch. d. byz. Litt.? S. 663 ff. unter Anführung einer beträchtlichen Anzahl von Gründen sich für Anastasios I (a. 491-518) entschieden hatte, ist er nunmehr geneigt, denen sich anzuschliefsen, welche unter dem Kaiser Anastasios den zweiten dieses Namens (a. 713-15) verstehen und „mit der Voraussetzung, dafs er ein Autor des 8. Jahrhunderts sei, wie mit einer Thatsache zu rechnen". Diese Sinnesänderung Krumbachers ist wesentlich eingetreten infolge der Interpretation von Anspielungen auf Zeitereignisse, die sich in einem Gedichte des Romanos finden, die aus einem Briefe Gelzers mitgeteilt wird. „Alle übrigen Argumente zu Gunsten des 6. Jahrhunderts beruhen auf mehr oder weniger wahrscheinlichen Kombinationen, können aber vor der positiven Thatsache einer deutlichen Anspielung auf Siege der Araber nicht standhalten" (p. 150). Demnach sollte man annehmen, dafs im Gegensatze zu jener stattlichen Reihe von „Wahrscheinlichkeiten", die bisher Krumbachers Urteil bestimmten, Gelzers Argumente von einer geradezu überwältigenden, jeden weiteren $\mathrm{Zweifel}$ ausschliefsenden Beweiskraft seien, und Gelzer selber schreibt sie ihnen jedenfalls zu, da er mittlerweile (Abhandl. der phil.-hist. Klasse der Sächs. Ges. d. W. Bd. 18 No. V p. 76) die Hauptblüte des Romanos "gerade in die eiserne Zeit des Kopronymos verlegt" und daran allgemeinere Betrachtungen über den Geist jener Zeit anknüpft, ohne seinen Lesern auch nur anzudeuten, dafs sich um die Bestimmung der Lebenszeit des Dichters eine schwierige und vielseitige Kontroverse dreht. Daher möchte ich meinen bei erster flüchtiger Lektüre sofort entstandenen und bei späterer Überlegung verstärkten Zweifeln an der Stichhaltigkeit der Behauptungen Gelzers Ausdruck geben. 
Romanos klagt über die schreckenvollen Ereignisse seiner Zeit; neben einer Reihe von Anspielungen auf furchtbare Naturereignisse und innere Mifsstände, wie sie zu allen Zeiten der byzantinischen Geschichte berechtigt waren, wird als Ausgangspunkt für eine sichere

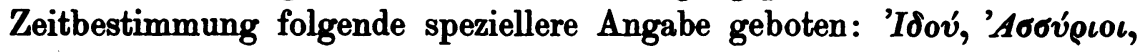

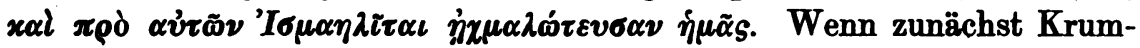
bacher hierzu bemerkt, Ismaeliten sei in byzantinischer Zeit die übliche Bezeichnung für die Araber gewesen, so ist das in dieser Form nicht richtig, da diese rom Vater abgeleitete Namensform ganz verschwindet neben der Ableitung von dem Namen der Mutter; soweit nicht die alte Bezeichnung $\Sigma \alpha \rho \alpha x \eta v o i$ beibehalten wird, ist durchweg üblich, sie

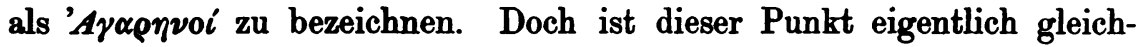
giltig, da es dem Dichter natürlich zu allen Zeiten freistand, die Nachkommen Ismaels als Ismaeliten zu bezeichnen, wenn ihm dieser Ausdruck gerade seiner seltneren Verwendung wegen poetischer erschien oder er ihm besser ins Versmals pafste. Gelzer erklärt nun kurzerhand:

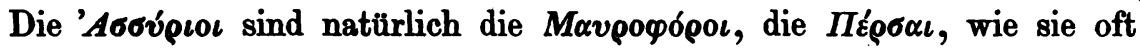
heifsen, die Kalifen ron Bagdad, die Abassiden und übersetzt: Sieh!

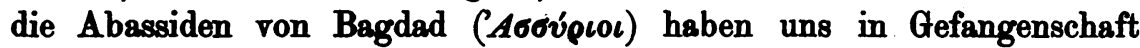

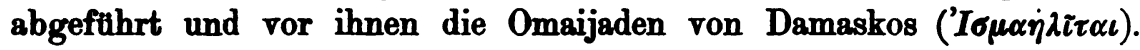
Aber die Abassiden, die Kalifen von Bagdad, waren doch keine Mavoo-

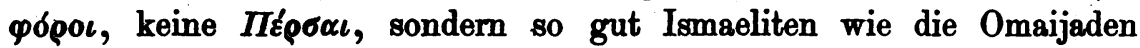
von Damaskos. Natürlich konnte der Dichter von der Lage der Residenz im Gebiete der alten Babylonier und Assyrier ausgehend die

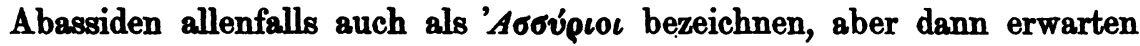
wir auch für die Omaijaden ein lokales oder sonstwie sie eigentümlich bezeichnendes Wort, nicht ein ethnographisches, welches die gegensätzlich Genannten mit umfafst. Im Gegensatz zu den Ismaeliten fafat

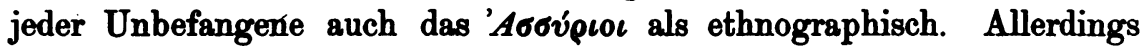
spielte ja das persische Element in den Bürgerkriegen, welche den Sturz der Omaijaden und die Verlegung der Residenz nach Bagdad herbeifuthrten, eine hervorragende Rolle und beeinflufste dauernd den Charakter desselben in mafsgebender Weise; es fragt sich nur, ob dieser Gegensatz in Byzanz je so empfunden worden ist, dafs von einem Schriftsteller diese Wesensverschiedenheit hervorgehoben und von seinen Lesern verstanden werden konnte, zumal so unmittelbar zur Zeit des Umschwungs selber - denn bis erheblich später läfst sich die Lebens- und Schaffenszeit des Romanos nicht wohl herabrücken. Aber nur bei einem Schriftsteller findet sich ein deutlicher Einblick in diese Verhältnisse, bei Theophanes, oder vielmehr nicht bei diesem, sondern bei dem Verfasser der Quelle, die dieser ausschrieb, 
und dieser Autor safs nicht in Byzanz, sondern offenbar in Syrien oder dessen Nähe, war also in der Lage und hatte ein Interesse daran, die verschiedenen Faktoren, die in diesen innern Kämpfen mit einander rangen, zu unterscheiden. Aber auch bei ihm treten, nachdem die Bürgerkriege beendet und die Angriffe auf Byzanz wieder aufgenommen sind, die Maurophori (die hier p. 452, 5; 456, 4; 482, 2 nur so, ohne

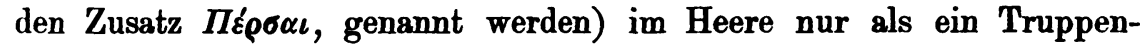
korps neben den übrigen Kontingenten aus Syrien, Mesopotamien und andern Provinzen auf, der Kalif aber wird nach wie vor als

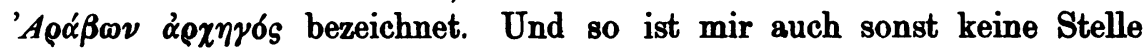
erinnerlich, welche darauf hinwiese, dafs irgend jemand in Byzanz bei dem Feinde, der auf demselben Wege, wie die Omaijaden, und unter derselben Frahne des Propheten ins Reich einfiel, einen Unterschied gegen die fruhhere Zeit gefunden hätte, weil das Zentrum, von dem der Angriff ausstrahlte, ein andres geworden war. Die Feinde bleiben dem Volksgeftuhl nach wie vor Araber, Sarazenen, Agarener, d. h. Söhne Ismaels.

Und ebensowenig wahrscheinlich erscheint mir die Beziehung der Ausdrücke des Gedichts auf jene Zeit, selbst zugegeben, dafs die

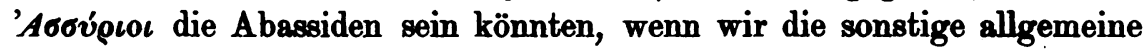
Weltlage in Betracht ziehen. Kam Romanos während der Regierung des Anastasios II nach Byzanz; so hatte er kurz darauf die Omaijaden vor den Thoren der Stadt, das Reich am Rande des Abgrunds gesehen, und es ist sehr begreiflich, wenn ihm selbst noch 40 Jahre später die Ismaeliten in schreckenvoller Erinnerung stehen. Aber dann war, wie Gelzer selbst angiebt, für lange Zeit der Arm, der bereits Byzanz zu zerschmettern gedroht hatte, gelähmt durch die innern Kriege der Araber; Kleinasien genofs eine längere Periode der Ruhe, und die Byzantiner konnten nicht nur die Raubzüge ihrer Feinde gründlich wettmachen, sondern längst verlorene Provinzen dem Reiche wieder angliedern. Freilich nur für wenige Jahre. Die Provinzen gingen bald wieder verloren, als durch die Abassiden die gesamte Kraft des Islam wieder zusammengefalst war; aber bedeutende sonstige Erfolge errangen diese, von den Chazaren und Türken schwer bedrängt, erst viel später, und Plünderungszüge in die Grenzprovinzen, die von den Byzantinern mit gleicher Münze bezahlt wurden, sind lange Zeit das Einzige, von dem wir hören. Ist es glaublich, dafs diese ersten Anzeichen eines beginnenden Rückschlags nach langer, ruhiger und glücklicher Zeit, diese wechselnden kleinen Erfolge an den äufsersten Grenzen, den Dichter sofort so erschüttert haben sollten, dafs er sie, wie jene Züge der Omaijaden, die die Ungläubigen zu Lande und zur See vor die 
Mauern von Byzanz geführt, als öffentliche Kalamität beklagte? Und das zu einer Zeit, wo den Bürgern von Byzanz andere Sorgen so viel näher lagen? Wenn auch freilich Kopronymos den Bulgaren wiederholt schwere Niederlagen beigebracht, so brachen sie doch immer wieder von neuem hervor und verwüsteten nicht weit entfernte Grenzprovinzen, sondern die nächste Gegend vor den Thoren der Stadt, den Landbesitz der Bürger von Byzanz. Wenn man an die Rolle der Bulgaren im 8. Jahrhundert denkt und bedenkt, wie natürlich, ja selbstverständlich es war, diese Reichsfeinde des Nordens und Westens denen des Südens und Ostens gegenüberzustellen, wenn man über die schweren Zeiten klagte, so tritt der Gegensatz zwischen Ismaeliten und Assyriern doppelt scharf in seiner Unnatürlichkeit und Künstelei hervor.

Somit kann ich in den Worten des Dichters nicht den mindesten Beweis für den Ansatz seiner Lebenszeit auf das 8. Jahrhundert finden; im Gegenteil, wenn wir sicher wülsten, dafs er jener Zeit angehörte, würden sie mich befremden und mir einer Erklärung bedürftig erscheinen. Trotzdem würden wir uns zu dieser Annahme bequemen müssen, wenn sich nachweisen liefse, dafs jene Anspielungen auf die Zeit des Anastasios I und seiner Nachfolger absolut nicht pafsten.

„Dafs die Ismaeliten nicht in das 6. Jahrhundert passen, hat schon Pitra richtig erkannt" (Krumb. a. a. 0. p. 143). Gewifs waren sie damals keine das byzantinische Reich bedrohende Weltmacht; aber wer die Schriftsteller jener Zeit liest, begegnet oft genug auch damals dem

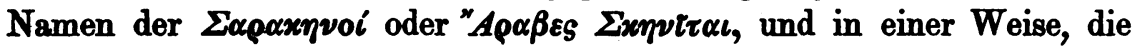
es durchaus verständlich machen würde, die Beduinenstämme unter den öffentlichen Kalamitäten des Reichs aufgeführt zu sehen, und auch die eigentümliche Art des Ausdrucks - früher die Ismaeliten, dann die Perser - findet in den historischen Verhältnissen ihre Rechtfertigung. Diese Wüstenbeduinen spielten durch die Lage ihrer Wohnsitze zugleich an der Grenze des persischen und des römischen Reiches eine eigentümliche Rolle. Da sie sich durch ihre schnelle Beweglichkeit

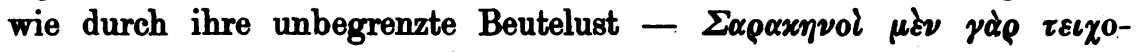

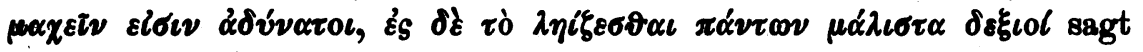
Prokop Pers. II 19 - als Feinde ebenso gefürchtet, wie als Freunde. nützlich zu machen wufsten, liefsen sich beide Mächte angelegen sein, sie durch Furcht in Zaum zu halten oder durch Geld zu gewinnen, und so standen schliefslich einige Stämme zu dieser, andre zu jener unter eigenen Stammesfürsten in einer Art von Abhängigkeitsverhältnis, sodals man von römischen und persischen Sarazenen sprach. Aber gerade in der Unbestimmtheit dieser halb abhängigen, halb selbständigen Stellung lag einer der Gründe zu der unverhältnismäfsig 
wichtigen Rolle, welche diese Sarazenen spielten, da sie für die diplomatischen Beziehungen zwischen den beiden Staaten eine immerwährende Gefahr bildete. Als Chosrau, besorgt wegen der grofsen Erfolge Justinians im Westen, nach Vorwänden sucht, um den Frieden zu brechen, reizt er den Phylarchen der persischen Sarazenen zu Einfällen in das Gebiet seiner römisch gesinnten Stammesgenossen und der Römer selbst und legt ihm die Behauptung in den Mund, die Friedensverträge zwischen den Römern und Persern bänden die Sarazenen nicht, da sie in denselben überhaupt nicht genannt seien. Letztere Thatsache giebt Prokop (Bell. Pers. II 1, Vol. I p. 154) zu, erklärt sie aber seinerseits dadurch, dafs die Sarazenen eben (als Unterthanen) unter den Namen der Römer und Perser einbegriffen seien. Für die Zeit Justinians mag er darin recht haben, dals das UnterthänigkeitsVerhältnis jener Stämme hinreichend straff gehandhabt worden sei, um einen Friedensvertrag zwischen beiden Reichen selbstrerständlich und ohne besondere Erwähnung auf sie-auszudehnen. Wenn er aber be-

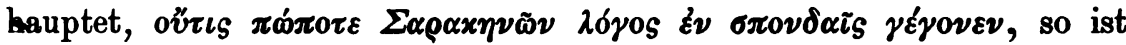
diese Angabe durchaus unzutreffend, denn in der früheren Zeit finden wir sie ganz wie eine selbständige Macht Krieg führend und Frieden schliefsend. Theodoret sagt über die Volksmengen, welche der Säule des Symeon Stylites zuströmten (Relig. Hist. c. 26, Migne tom. 82

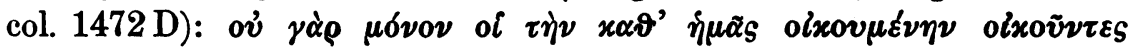

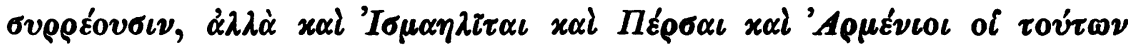

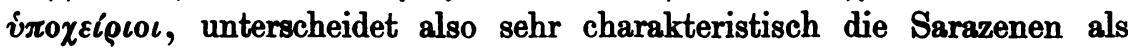
selbständige Nation neben den Persern von den unter diesen als Unterthanen stehenden Armeniern. Und während seit der Regierung des Theodosios II zwischen Römern und Persern Friede herrschte, verwüsteten die Araber unter Zenon den Osten (Euagr. III 2; Theoph. p. 120,10), und in der ersten Hälfte der Regierung des Anastasios I, ebenfalls vor Wiederausbruch des Perserkrieges, führten sie mit wechselndem Erfolge jahrelange erbitterte Kämpfe mit den Römern. Während sie (im 7. Jahre des Kaisers nach Theoph. p. 141, 1 ff.) nach Verwüstung von Mesopotamien, beider Phönizien und Palästina (Euagr. III 36) überall zurückgeschlagen wurden, gelingt ihnen (im 11. Jahr des Kaisers nach Theoph. 143, 21) ein neuer Raubzug in denselben Gegenden, ohne dafs es den römischen Feldherrn gelungen wäre, die wie ein Sturmwind einbrechenden Beduinen $\mathrm{zu}$ erreichen. Im folgenden Jahre (Theoph.

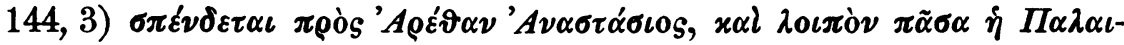

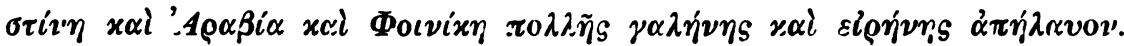
Dieser Friedensschlufs wird nicht nur aus gleicher Quelle von Euagrios bestätigt, sondern auch ron Nonnosos (Photios Bibl. cod. 3), dessen Byzant. Zeitschrift IX 4. 
Grofsvater als Gesandter den Vertrag abschlofs. In der That hören wir längere Zeit nichts mehr von Plünderungszügen der Sarazenen, und erst, als unter Justin I der Perserkrieg wieder ausgebrochen war, erscheinen sie wieder, aber in der ganzen nächstfolgenden Zeit als Hilfsvölker der Perser. Nach Theoph. p. 170, 30 (Mal. p. 423, 23) ernennt dieser Kaiser den Hypatios zum Oberfeldherrn im Orient, $\varphi v \lambda \alpha \alpha^{\prime} \xi \iota \iota \tau \grave{\alpha}$

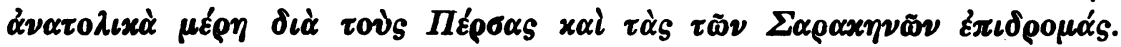
Damit hängt offenbar zusammen die nach der Thronbesteigung Justinians sofort in Angriff genommene Neubefestigung von Palmyra und die stärkere Besetzung der Militärgrenze gegen die Araber (Mal. p. 426, 1; Theoph. p. 174, 11). Bald darauf wird die gesamte Truppenmacht der Grenzprovinzen Arabien, Phönizien und Mesopotamien aufgeboten und ein siegreicher Zug bis tief in das Gebiet der feindlichen Sarazenen unternommen (Mal. p. 434, 19; Theoph. p. 179, 15). Doch bald erscheint der Phylarch Alamundaros wieder mit persischen und sarazenischen Völkern und plündert Syrien bis nahe an die Thore von Antiochia (Mal. p. 445, 1; Theoph. 178, 7), später Osrhoëne (Mal. p. 461, 8). Doch war der Krieg, da es gelang, die Hsuptmacht der Perser in Schach zu halten, ubberhøupt schwerlich ein Gegenstand besondrer Sorge und Erregung für Byzanz. Um so mehr wird dies der Fall gewesen sein, als im Jahre 540 sich unter die Siegesbotschaften aus dem Westen die fatale Kunde mischte, dafs Chosrau von neuem die Fahne des Krieges erhoben, und bald eine Hiobspost nach der andern die Residenz erreichte. Diesmal waren es nicht die Sarazenenschwärme, die auf flüchtigem Raubzug Syrien verwisteten, sondern das persische Heer selbst, welches unter Führung des Königs weite Strecken des Römerreichs widerstandslos brandschatzte und plünderte, die Städte, voran Antiochia, zeratorite oder mit unerhörten Kontributionen belegte und ganee Finwohnerschaften wegschleppte und im Perserreich ansiedelte. Im folgenden Jahre erscheint der Perserkönig erfolgreich in den nordöstlichen Provinzen des Reiches. Diese Lage war um so gefährlicher, als die Hauptkräfte des Reichs noch im Westen beschäftigt waren und durch mehrjährigen Kampf Heer und Finanzen stark in Anspruch genommen waren.

Ubbersieht man diese Zustände und diese Entwickelung der Verhältnisse an der Ostgrenze - und auf diese allein, an der der unermuldliche Erbfeind sals, konnten die Blicke sich damals mit Sorge richten -, so sieht man, wie mit vollem Rechte der Dichter sagen konnte: Früher (als der lange Friedenszustand mit den Persern herrschte) plünderten uns die Sarazenen aus, jetzt die Perser selber. Die selbständigen Kriegszulge der Sarazenen gegen Syrien in der ersten Hälfte 
der Regierung des Anastasios kaun Romanos noch selbst in seiner Heimat erlebt haben und einen um so tiefern und dauernderen Eindruck davon bekommen haben, und wiederum gerade ganz besonders seine Heimatprovinz wurde von den Zügen Chosraus aufs fürchterlichste betroffen. Dals die feueranbetenden Perser und die heidnischen Sarazenen, welche die Aphrodite verehrten und ihr Menschenopfer brachten (Theodoret, Relig. Hist. c. 26; Prokop Pers. II 26; Euagr. VI 22), nicht nur als Reichsfeinde, sondern auch als Gottes Feinde bezeichnet werden, versteht sich von selbst.

Dals den Byzantinern jener früheren Zeit die Herleitung dieser Beduinenstämme von Ismael ganz geläufig war, beweist die von Gelzer angeführte Stelle des Hieronymus. Ähnlich sagt Theodoret in Ieremiam

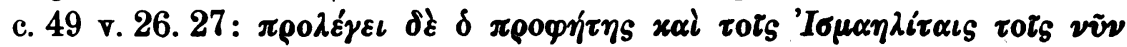

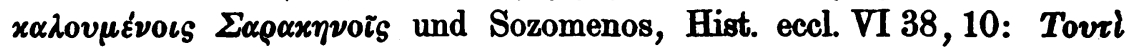

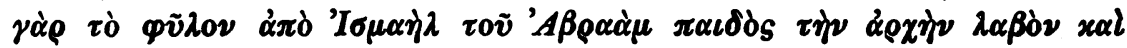

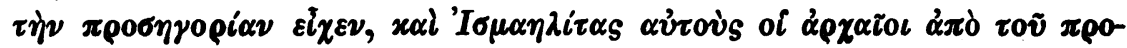
$\pi \dot{\alpha} \tau 0 \rho \circ \dot{\omega}^{2} \nu \sigma \alpha \alpha \sigma \alpha \nu$. Dafs aber nicht nur die $\alpha \rho \chi \alpha \tau o c$ sie so nannten, sondern dieser Name auch später noch, wenigstens in den Grenzprovinzen, in Gebrauch war, dafür ist Theodoret Zeuge, der mit Vorliebe davon Gebrauch macht. Ich zitierte oben bereits eine Stelle der Historia religiosa c. 26; im weitern Verlaufe des Kapitels finden wir

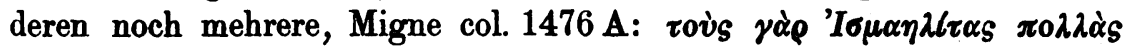

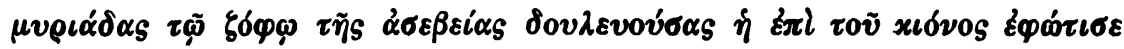

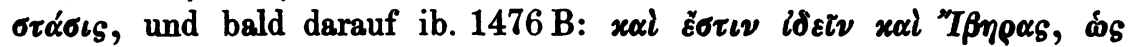

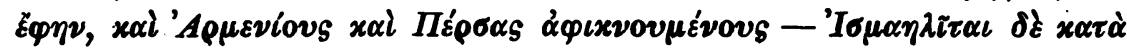

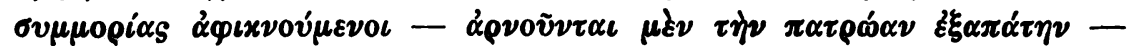

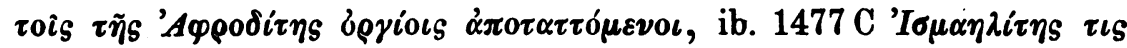
oủx $\ddot{\alpha} \sigma \eta \mu o s$. Und Graec. aff. cur. libr. V (Migne tom. 83 col. $949 \mathrm{D}$ ):

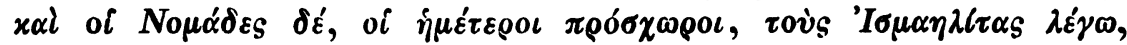

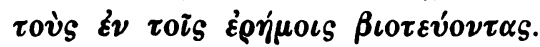

Dafs die sonstigen Anspielungen auf das 6. Jahrhundert ebenso gut passen wie auf das achte, ist bekannt. Wie Krumbacher für dieses eine Stelle des Theophanes aus dem Jahre 740 anführt, so möchte ich auf das Kapitel des Georgios Monachos (p. $538 \mathrm{ff}$. ed. Muralt) hinweisen, in dem er alles zusammenstellt, was er in seinen Quellen über solche Naturereignisse und innere Schrecknisse während der Regierung Justi-

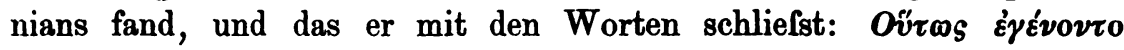

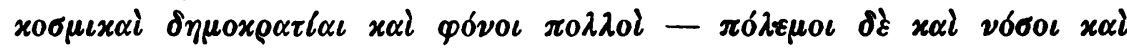

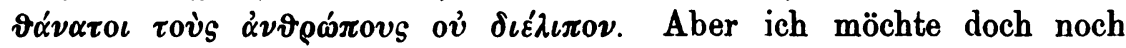
besonders darauf aufmerksam machen, wie sich gerade unmittelbar nach den furchtbaren Kerwüstungen der Perser solche Ereignisse anhäuften. 


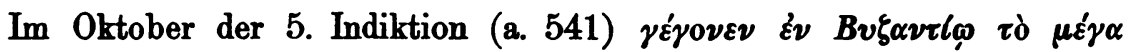

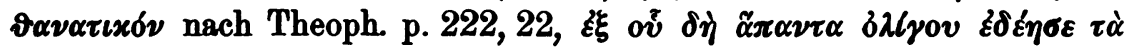

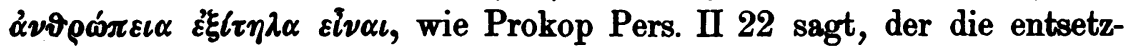
lichen Verheerungen der Pest ausführlich schildert. Im Anschlufs daran

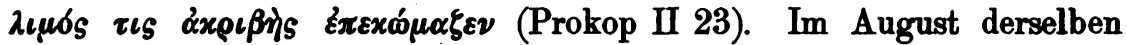
Indiktion (a. 542) erschütterte ein heftiges Erdbeben Konstantinopel,

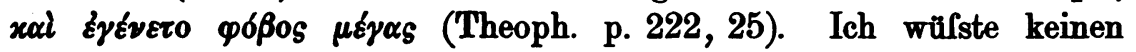
Moment der byzantinischen Geschichte, in dem die Klagen des Romanos in so erschütternder Weise der Wirklichkeit angepafst wären, wie im Herbste des Jahres 542.

Trotzdem liegt mir natürlich nichts ferner, als die Abfassungszeit des Gedichts auf dies Jahr zu fixieren, ja ich vermesse mich nicht einmal zu behaupten, mit meinen Ausführungen bewiesen zu haben, dafs die Lebenszeit des Dichters in das 6. Jahrhundert fällt. Das Problem ist zu mannigfaltig, um bei der Allgemeinheit der Ausdrücke des Dichters ausschliefslich aus diesem einen Gesichtspunkt gelöst zu werden. Was ich gewünscht habe nachzuweisen ist nur, dals diese Andeutungen keineswegs auf das 8 . Jahrhundert bezogen werden müssen, vielmehr sich ebenso gut, ja weit ungezwungener, aus den Verhältnissen des 6. Jahrhunderts erklären lassen. $O b$ man in der Entscheidung weiter kommen wird, wenn man die Dichtungen, in denen bisher niemand, wie es scheint, die geringste Anspielung auf die Bilderkämpfe entdeckt hat, unter die Lupe nimmt und dabei selbst einige Achabs und Herodes entdeckt, ob uberhaupt in jener wildbewegten Zeit solche von den Parteikämpfen unberührte Dichtungen denkbar sind, geschweige denn der Dichter der Dante einer solchen Zeit werden konnte, will ich hier nicht erörtern. „Es wird ja“, um mit Worten Krumbachers zu schliefsen, „unvermeidlich sein, später in gröfserem Zusammenhang noch einmal auf die ganze Frage zurückzukommen, wenn einmal der Nachlars des Dichters vollständig publiziert vorliegen wird“, und der Berufenste, wenn nicht einzig Mögliche, der dies Postulat erfüllen kann, ist eben der, der es aufgestellt hat.

Breslau.

C. de Boor. 